\title{
SCIENTIFIC REPORTS

\section{Immunosuppressive effect and global dysregulation of blood transcriptome in response to psychosocial stress in vervet monkeys (Chlorocebus sabaeus)}

\author{
Anna J. Jasinska $\mathbb{1}^{1,2^{*}}$, Ivona Pandrea $\mathbb{1}^{3}$, Tianyu $\mathrm{He}^{3}$, Cassandra Benjamin ${ }^{4}$, \\ Maurice Newton ${ }^{4}$, Jen Chieh Lee ${ }^{1}$, Nelson B. Freimer ${ }^{1}{ }^{1}$, Giovanni Coppola ${ }^{1}{ }^{1,5}$ \& \\ James D. Jentsch ${ }^{6}$
}

Psychosocial stressors - life events that challenge social support and relationships - represent powerful risk factors for human disease; included amongst these events are relocation, isolation and displacement. To evaluate the impact of a controlled psychosocial stressor on physiology and underlying molecular pathways, we longitudinally studied the influence of a 28-day period of quarantine on biomarkers of immune signalling, microbial translocation, glycaemic health and blood transcriptome in the wild-born vervet monkey. This event caused a coordinated, mostly transient, reduction of circulating levels of nine immune signalling molecules. These were paralleled by a massive dysregulation of blood transcriptome, including genes implicated in chronic pathologies and immune functions. Immune and inflammatory functions were enriched among the genes downregulated in response to stress. An upregulation of genes involved in blood coagulation, platelet activation was characteristic of the rapid response to stress induction. Stress also decreased neutrophils and increased $\mathrm{CD} 4+\mathrm{T}$ cell proportions in blood. This model of psychosocial stress, characterised by an immune dysregulation at the transcriptomic, molecular and cellular levels, creates opportunities to uncover the underlying mechanisms of stress-related diseases with an immune component, including cardiovascular diseases and susceptibility to infections.

Psychosocial stress is a key risk factor for numerous chronic diseases, particularly cardiovascular (e.g., hypertension and atherosclerosis), metabolic (e.g., diabetes), and neuro-psychiatric morbidity (e.g., depression and anxiety, substance use disorders, neurodegenerative disease and psychosis $)^{1-8}$. Stress responses constitute an adaptive strategy for maintaining bodily homeostasis in physically or psychologically challenging situations and are, in part, orchestrated by the hypothalamus-pituitary-adrenal (HPA) axis and controlled by its end products, glucocorticoids, through a negative feedback loop. However, prolonged exposure to stress, chronic activation of the HPA, and continuous secretion of glucocorticoids exert maladaptive effects and lead to the wide range of health complications mentioned above. Cortisol is regarded as a major mediator of a range of stress responses affecting many body systems, including the suppression of HPA activity, regulation of immune functions, glucose metabolism, and gene expression across different organ systems and, if chronically elevated, is a key driver of stress-related pathologies.

Cortisol is commonly used physiological indicator of an individual's response to stress; ${ }^{9,10}$ notably, however, this relationship is moderated by multiple biological and environmental variables (including - but not limited to

\footnotetext{
${ }^{1}$ Center for Neurobehavioral Genetics, Semel Institute for Neuroscience and Human Behavior, The University of California Los Angeles, California, USA. 'Institute of Bioorganic Chemistry, Polish Academy of Sciences, Poznan, Poland. ${ }^{3}$ Department of Pathology, School of Medicine, University of Pittsburgh, Pittsburgh, Pennsylvania, USA. ${ }^{4}$ St. Kitts Biomedical Research Foundation, St. Kitts, West Indies, Saint Kitts and Nevis. ${ }^{5}$ Department of Neurology, The University of California Los Angeles, California, USA. ${ }^{6}$ Department of Psychology, Binghamton University, Binghamton, NY, 13902, USA. *email: ankajasinska@gmail.com
} 
- age, circadian rhythm, nutritional status and other exposures) and the tissue or other biological resource used for measurements ${ }^{11-15}$. Additionally, the magnitude of the cortisol response to stress can habituate or sensitize with repeated or prolonged exposure to the same or different stressors, effects mediated through multiple mechanisms, including altered negative feedback at the level of brain and pituitary, changes in the activity of pro-stress circuitry in brain, and complex learning mechanisms ${ }^{16,17}$. Consequently, the interpretation of cortisol levels as biomarker of chronic stress should therefore be treated with caution.

Importantly, not all individuals respond equivalently to the same stress challenge, nor do they show the same stress-induced pathology. Inter-individual variability in stress responsivity and susceptibility to stress-triggered diseases is shaped by multiple factors, including the variety of stressors encountered in everyday life, other environmental factors, and comorbid disorders. These confounding variables are difficult to control or even account for (such as diet, medications, lifestyle, etc.) in human cohorts. Many of these factors could be better controlled in an experimental setting using animal models, among which Non-Human Primates (NHPs) stand out as most closely reproducing the biology of the cognitive, metabolic, and immune processes observed in humans because of their close phylogenetic relatedness ${ }^{18}$.

In naturally living NHPs, a major form of psychosocial stress derives from the major rearrangements in social support and hierarchy that often occur across the lifespan ${ }^{19}$. In a laboratory setting, some of these stressful events can be mimicked when animal relocation between facilities and/or social group rearrangements occur, creating a model system for studying responses to a defined psychosocial stress. Relocations were previously associated with increased cortisol levels in Garnett's bushbaby ${ }^{20}$ and with behavioral consequences such as self-injuries and sleep disturbances in rhesus macaques ${ }^{21}$, demonstrating that they can trigger both neuroendocrine and behavioral stress responses in NHPs serving as a model for stress studies. Relocation has also been observed to affect hematological, clinical chemistry, and immunological parameters in chimpanzees ${ }^{22}$. Psychosocial stressors in NHPs, such as experimentally enforced low position in a social hierarchy/dominance rank, have lasting yet plastic (i.e., reversible) effects on gene expression in blood and also affect blood cell composition, diminish negative feedback on the HPA, and modulate chromatin accessibility ${ }^{23,24}$. Overall, investigations involving removal from a current group, experimental manipulation of social rank, and early life adversities (e.g., adverse prenatal environment and early postnatal rearing environments) demonstrated both that the NHPs share many characteristics of the stress response with humans ${ }^{25}$ and that social environment has an effect on health and gene expression in both humans and $\mathrm{NHPs}^{26,27}$.

To develop an NHP model of psychosocial stress (PS) exposure that would facilitate longitudinal studies of the effects of a uniform (standardized) stressor under controlled conditions, we turned to the vervet monkey (Chlorocebus sabaeus), an established NHP biomedical research model ${ }^{28-30}$. The vervet represented an ideal animal model for our study as it was previously reported to show significant neuroendocrine and behavioral responses to relocation between research centers ${ }^{31,32}$ and neuroanatomical alterations (in the hippocampus) upon relocation from the wild to a research facility ${ }^{33}$.

A small number of vervets from West Africa were introduced to the Caribbean Islands during the colonial era. The feral vervet population vastly expanded in the ecosystem due to the absence of natural predators. Wild-born vervet monkeys from the highly abundant population on St. Kitts Island are currently a source of animals for biomedical research in multiple local primate facilities ${ }^{28}$. After capture, wild-born monkeys are placed in a 28 -day quarantine in the primate facility, during which they are sometimes housed individually and assessed for infectious agents and behavioral problems before being integrated to the colony. We hypothesized that the temporary disruption of social support following removal from the family troop and placement in solitary housing would be a meaningful psychosocial stressor that would exceed other stressors experienced thus far in juvenile individuals and, therefore, that this setting would represent a natural model for stress studies.

Here, we present the neuroendocrine, immune, metabolic, and transcriptomic responses of 15 Kittitian juvenile vervet males subjected to conditions closely mimicking the standardized quarantine procedures. We demonstrate that the quarantine-related stress significantly dysregulated the immune system, blood transcriptome, and blood cell composition, supporting our hypothesis that the quarantine setting represents a natural model system for studies on PS in the vervet monkey.

\section{Results}

Biochemical responses to PS. During the period of relocation and quarantine, hair cortisol increased from $68.62 \pm 83.03 \mathrm{pg} / \mathrm{mg}$ (before quarantine at day 0 (Tp1)) to $96.49 \pm 62.65 \mathrm{pg} / \mathrm{mg}$ (after quarantine on day 28 (Tp6)) (Supplementary Figure 1). A Wilcoxon Signed-Rank Test indicated that hair cortisol level was statistically significantly increased after the quarantine $Z=1.69, \mathrm{p}<0.0455$ (one tail), when the entire group of 15 individuals was tested. We observed large inter-individual variability in baseline cortisol levels because of two individuals, VSC00010 and VSC00014, that exhibited high baseline cortisol levels. These individuals came from different regions of St. Kitts Island and did not show any visible signs of old or fresh injuries, poor nutrition, or other health problems that could explain the elevated cortisol levels or additional sources of stress. It is possible that these individuals experienced unknown social circumstances in their troops ${ }^{34}$ that contributed to their high cortisol levels. After quarantine, there were two upper outliers that stood out due to very high hair cortisol levels: VSC00010 and VSC00015 (which was the only individual subjected to quarantine during Hurricane Danny, a possible additional stressor). After exclusion of these three individuals with outlying individuals measurements (defined according to the $1.5 \mathrm{x}$ IQR rule), we observed a significant increase in hair cortisol level from $39 \pm 15.96 \mathrm{pg} / \mathrm{mg}$ on day 0 (Tp1) to $72.10 \pm 30.69 \mathrm{pg} / \mathrm{mg}$ on day 28 (Tp6) after the quarantine (Wilcoxon Signed-Ranks test, $\mathrm{Z}=2.57, \mathrm{p}<0.0051$ [one tail]). This increase in cortisol during quarantine is consistent with the sustained activation of the HPA axis, implying that quarantine was a meaningful stressor and thereby demonstrating the validity of our PS model.

Stress can induce a wide spectrum of alterations in immune indices associated with immunoprotective, immunopathological, and immunoregulatory/inhibitory effects ${ }^{35}$. Longitudinal assessment of circulating levels of 29 


\begin{tabular}{|c|c|c|c|}
\hline Variable & $\begin{array}{l}\text { ANOVA } \\
\text { p-value }\end{array}$ & $\begin{array}{l}\text { Tp6 vs. Tp1 } \\
\text { p-value* }\end{array}$ & $\begin{array}{l}\text { p-value for inclusion } \\
\text { of random effect }\end{array}$ \\
\hline sCD14 & 4.14E-01 & $6.00 \mathrm{e}-01$ & $1.06 \mathrm{E}-01$ \\
\hline LPS & 4.19E-01 & $8.05 e-02$ & $2.26 \mathrm{E}-01$ \\
\hline IL- $1 \beta$ & \begin{tabular}{|l|}
$8.49 \mathrm{E}-05$ \\
\end{tabular} & $5.36 \mathrm{e}-01$ & \begin{tabular}{|l|}
$1.17 \mathrm{E}-04$ \\
\end{tabular} \\
\hline IL-12 & 2.41E-01 & \begin{tabular}{|l|}
$9.18 \mathrm{e}-01$ \\
\end{tabular} & \begin{tabular}{|l|}
$1.33 \mathrm{E}-07$ \\
\end{tabular} \\
\hline RANTES & 1.67E-04 & 6.41e-01 & 5.11E-04 \\
\hline Eotaxin & 2.07E-03 & $1.18 \mathrm{e}-03$ & 3.22E-03 \\
\hline MCP-1 & \begin{tabular}{|l|}
$8.24 \mathrm{E}-06$ \\
\end{tabular} & 5.41e-04 & \begin{tabular}{|l|}
$9.76 \mathrm{E}-03$ \\
\end{tabular} \\
\hline HGF & 3.75E-05 & 4.02e-02 & $8.74 \mathrm{E}-06$ \\
\hline IFN- $\gamma$ & 3.48E-05 & $1.65 \mathrm{e}-02$ & \begin{tabular}{|l|}
$3.56 \mathrm{E}-04$ \\
\end{tabular} \\
\hline MDC & \begin{tabular}{|l|}
$4.46 \mathrm{E}-04$ \\
\end{tabular} & \begin{tabular}{|l|}
$3.14 \mathrm{e}-02$ \\
\end{tabular} & \begin{tabular}{|l|}
$2.74 \mathrm{E}-02$ \\
\end{tabular} \\
\hline MIF & \begin{tabular}{|l|}
$1.73 \mathrm{E}-09$ \\
\end{tabular} & 3.53e-04 & \begin{tabular}{|l|}
$2.40 \mathrm{E}-05$ \\
\end{tabular} \\
\hline IL-1RA & $1.50 \mathrm{E}-10$ & 2.25e-06 & \begin{tabular}{|l|}
$4.33 \mathrm{E}-05$ \\
\end{tabular} \\
\hline IP-10 & 5.36E-01 & $6.20 \mathrm{e}-01$ & \begin{tabular}{|l|}
$2.18 E-03$ \\
\end{tabular} \\
\hline IL-4 & 3.30E-01 & \begin{tabular}{|l|}
$1.870 \mathrm{e}-01$ \\
\end{tabular} & 4.83E-01 \\
\hline Alc & 1.26E-01 & $3.11 \mathrm{e}-01$ & \begin{tabular}{|l|}
$1.97 \mathrm{E}-02$ \\
\end{tabular} \\
\hline GHb & $1.44 \mathrm{E}-01$ & $2.09 \mathrm{e}-01$ & $2.76 \mathrm{E}-02$ \\
\hline
\end{tabular}

Table 1. Variability of immune, microbial translocation, and glycemic biomarkers. bold face p-value $<0.05$. *paired t-test p-value.

immune biomarkers revealed that mean levels of nine molecules (IL-1 $\beta$, IL-1RA, IFN- $\gamma$, eotaxin, MCP1, MDC, MIF, RANTES, and HGF) showed changes across the experimental time points that were nominally significant $(\mathrm{p}<0.05$, Table 1$)$. Immune biomarker molecules with typically pro-inflammatory effects (e.g., IL- $1 \beta$ and IFN- $\gamma$ ) as well such molecules with anti-inflammatory effects (e.g., IL1-RA and HGF) showed a coordinated decrease in response to the relocation and quarantine stressor (Fig. 1). The gradual decrease in average cytokine levels, which reached their maximum on day 14, is consistent with PS-induced suppression of the immune system. After day 14, average levels of RANTES and IL- $1 \beta$ evidenced recovery, suggesting progressive habituation to the stressor, while seven other biomarkers (Eotaxin, MCP-1, HGF, IFN- $\gamma$, MDC, MIF, IL-1RA) remained significantly different $(\mathrm{P}<0.05)$ from the baseline at the end of quarantine period. Stress can induce immunoprotective (e.g., promote wound healing and eliminate infections), immunopathological, and immunoregulatory effects depending on the context and the duration of the stressor ${ }^{36-40}$. Typically, short-term stress lasting for minutes, hours, or days triggers adaptive mechanisms, preparing the body for challenges that may be imposed by stressor, such as wounds, injuries, or infections, through enhancing immune responses. However, long-term or repeated, intermittent stress lasting for weeks or months can suppress or dysregulate immune responses ${ }^{38}$. Based on the pattern in immune marker response, we can conclude that the applied quarantine conditions represented a long-term, persistent stressor that evoked immune suppression.

Psychosocial stress can also increase the permeability of the intestinal barrier, causing the translocation of bacteria and antigens from the gut to the bloodstream and, in turn, activating pro-inflammatory responses ${ }^{41-43}$. To assess the potential effects of PS on the permeability of the gut epithelium and systemic inflammatory state, we investigated plasma levels of the microbial translocation biomarker sCD14, an indicator of monocyte activation, as well as levels of the bacterial endotoxin lipopolysaccharide (LPS). We did not observe significant changes in circulating levels of sCD14 or LPS (Supplementary Figure 2), suggesting that the animals maintained an intact mucosal barrier that prevented microbial translocation.

Given that emotional stress is a risk factor for the development of type 2 diabetes (T2D) in healthy individuals $^{44}$ and aggravates outcomes in people already affected by T2D ${ }^{45}$, we predicted that PS would increase circulating levels of the glycemic biomarkers, $\mathrm{A} 1 \mathrm{C}$ and $\mathrm{GHb}$. We observed that neither of these glycemic biomarkers showed significant variability over the course of the PS (Supplementary Figure 3). The lack of effect on these glycemic measures may be related to the young age of our study subjects, considering that the risk of type II diabetes generally increases with older age ${ }^{46,47}$. Also, the 28 -day exposure period may be too short to dysregulate the metabolic system and reflect only a fraction of the potential long-term alterations to hemoglobin glycation levels.

Individuals differ with respect to perception of and reaction to stressors, resulting in different susceptibilities to stress-related diseases. These differences are generally shaped by two factors: genetic background and environmental exposure. We anticipated that the well-controlled quarantine environment, where external influences were minimized, would make the inter-individual variability in stress responses due to genetic differences more apparent. We observed that, of nine stress-reactive immune markers, all - except IL- 4 - showed significant inter-individual variability in the stress response (Table 1) and may represent candidate biomarkers with potential genetic contribution to stress reactivity for further studies.

Massive dysregulation of blood transcriptome in response to the relocation stressor. Glucocorticoids have widespread effects on transcriptional regulation ${ }^{48}$. To assess stress-related changes in gene expression, we analyzed the RNA-seq transcriptomic profiles of the monkeys at three time points during the PS experiment: day 0 (Tp1, baseline conditions), day 3 (Tp2), and day 14 (Tp4). The latter two time points hypothetically represented short-term and long-term PS exposures, respectively. We employed two approaches to identify genes 
$\mathrm{IL}-1 \beta$

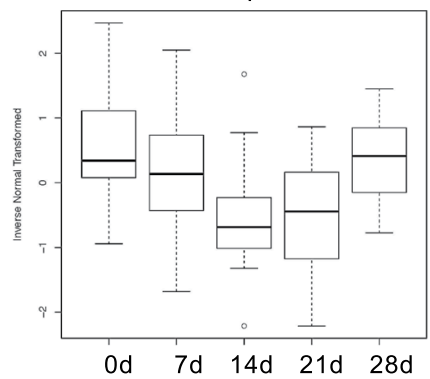

INF- $\gamma$

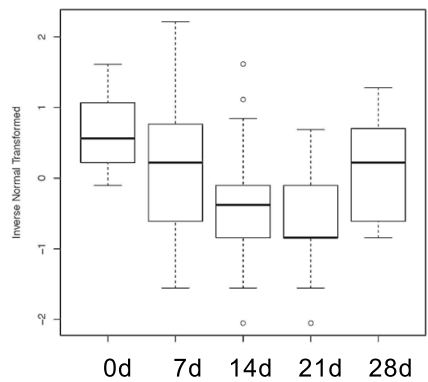

MCP1 - CCL2

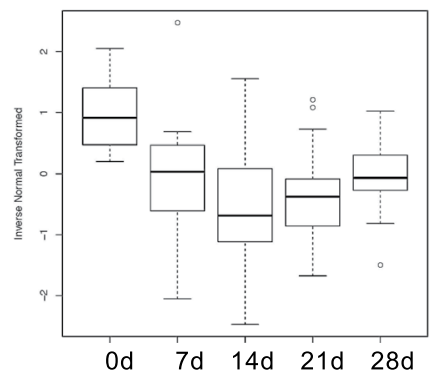

RANTES - CCL5

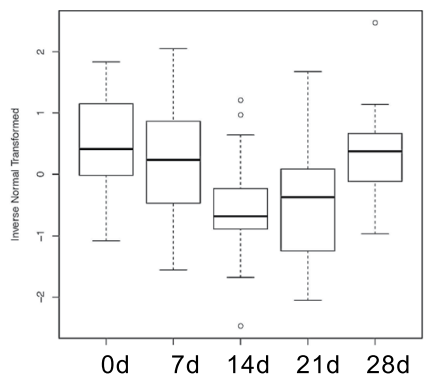

$\mathrm{MDC}-\mathrm{CCL} 22$

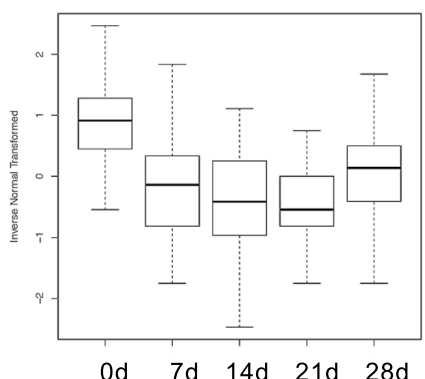

IL-1RA

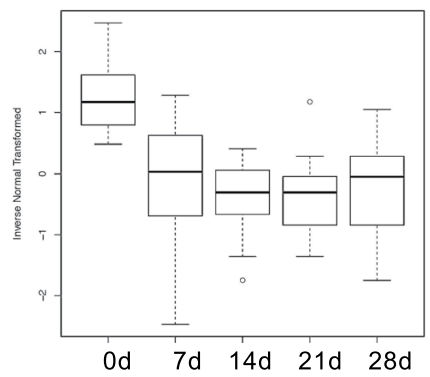

HGF

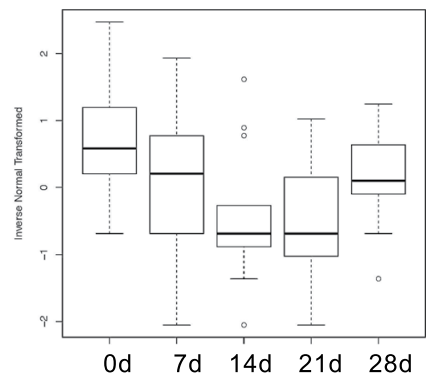

MIF

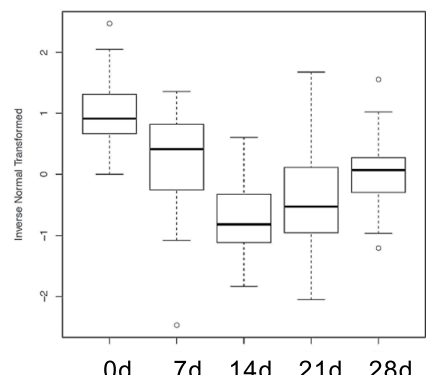

Od $\quad 7 d \quad 14 d \quad 21 d \quad 28 d$

Eotaxin - CCL11

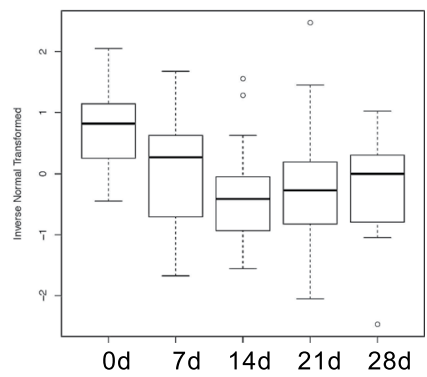

Figure 1. Circulating cytokine, chemokine, and growth factor levels (IL-1 $\beta$, RANTES, Eotaxin, MCP1, HGF, IFN- $\gamma$, MDC, MIF, IL-1RA) at five experimental time points: day 0 (Tp1 i.e. baseline), day 7 (Tp3), day 14 (Tp4), day 21 (Tp5), and day 28 (Tp6).

whose expression changed over time in response to the stressor: differential gene expression analysis, comparing expression between different experimental time points, and network analysis, identifying modules of co-expressed genes associated with experimental time points.

We observed significant changes in the expression of 3,393 genes on day 3 vs. day 0, 7,551 genes on day 14 vs. day 0 , and 4,286 genes on day 14 vs. day 3 (FDR threshold of 0.05), corresponding with a total of 9,055 dysregulated genes (Supplementary Table 1). Considering an absolute log2 fold change of "1" or greater as the threshold, we observed significant dysregulation in the expression of 556 genes (205 genes up-regulated and 351 genes down-regulated) on day 3 vs. day 0, 2,304 genes (1,441 up and 863 down) on day 14 vs. day 0 , and 863 genes (700 up and 163 down) on day 14 vs. day 3.

Many of the genes that showed a strong dysregulation in response to the PS ( $\log 2 \mathrm{FC}$ below -3 or above 3 ) are involved in biological processes related to different pathologies, including cardiovascular disease, Alzheimer's disease, tumor formation, oxidative stress, and neurotransmission. To assess which biological functions were impacted by the PS, we evaluated the possible enrichment of functional gene categories (GO and KEGG terms) among the dysregulated genes. Among the most strongly enriched GO biological processes in genes dysregulated on day 3 vs. the baseline were cell death, cellular responses to stress and chemical stimuli, and cell cycle regulation. On day 14 vs. baseline, the enriched GOs were cell cycle processes (and related processes) and cellular responses to stress (Fig. 2).

Considering the Kyoto Encyclopedia of Genes and Genomes (KEGG) pathways, the dysregulated pathways on day 3 vs. the baseline were enriched for the platelet activation pathway (hsa04611), the AMPK and MAPK signaling pathways, and cancer (Fig. 3). On day 14 vs. the baseline, among the most overrepresented KEGG pathways were those related to homeostatic processes, including HIF-1 signaling (involved in oxygen homeostasis and immunological response), insulin signaling (involved in energy homeostasis), and AMPK signaling (a key regulator of cellular energy homeostasis). Other enriched pathways were related to cancer, including acute myeloid leukemia, prostate cancer, and renal carcinoma, and Fc gamma R-mediated phagocytes, which are essential for host defense because of their participation in the uptake and destruction of infectious pathogens. 

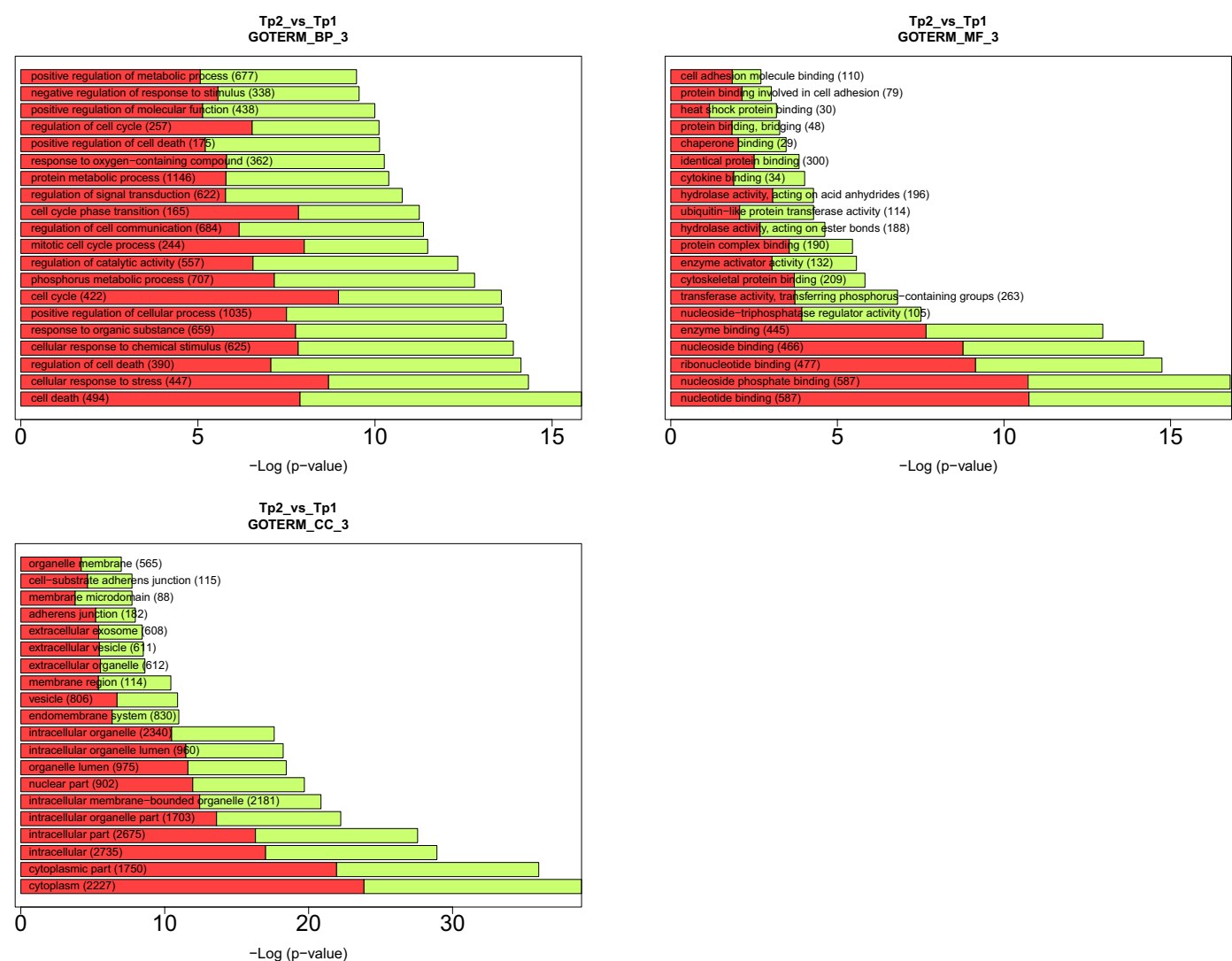

Figure 2. Gene Ontology (GO) enrichment analysis among stress-related genes genes based on the David tool. The red and green bars correspond to the proportion of up- and down-regulated genes.

Weighted Gene Correlation Network Analysis (WGCNA) of blood transcriptomic responses to PS. We grouped genes into modules using WGCNA (Supplementary Figure 4). We identified 31 gene modules, including 10 modules that were significantly correlated with at least one experimental time point (correl. $\geq 0.55$, p-value $<0.001$ ) (Fig. 4). We conducted pathway enrichment analysis for significantly correlated modules. The modules that showed the strongest correlations with a time point (correl. $\geq 65$ ) were associated with rather general biological terms. For example, the saddle brown module (correl. 71 with day 0 ) was associated with alternative splicing. The midnight module (correl. 69 with day 0 ) was associated with alternative splicing and phosphoprotein. The royal blue module (correl. 65 with day 15) was associated with protein modification. The black module (correl. 65 with day 15) was associated with acetylation, cell division, mitotic processes, and mitochondrial respiratory chain functions. Finally, the turquoise module (correl. -55 with day 0 and correl. 63 with day 15) showed increased expression during the PS experiment and enrichment of phosphoprotein, acetylation, and mitotic and cell cycle processes.

More specific biological terms were associated with the modules moderately and significantly correlated with the course of PS (Fig. 5). The blue module (correl. 56 with day 0) showed the enrichment of numerous terms related to immune and inflammatory functions, including the immune system process, defense response, immune response, response to stress, inflammatory response, apoptotic process, and cell death. These observations suggest that PS can impact health through the disruption of immune, inflammatory, and cell death processes. The dark green module (correl. 55 with day 3 ) was characterized by its transiently elevated expression on day 3; enrichment was observed for blood coagulation, platelet activation, wound healing, and response to wounding. This observation is consistent with the association of the platelet activation pathway with transient gene dysregulation on day 3 and points to increased cardiovascular risk following short-term PS.

Stress alters cellular composition of blood. Blood is a heterogeneous tissue comprised of a mixture of different cell subtypes. We investigated whether stress had an effect on the composition of cell types in peripheral blood. To estimate the proportions of different cell subsets in our whole blood samples, we employed deconvolution of the bulk transcriptomic data using the Cibersort tool ${ }^{49}$. We compared the estimated fractions of specific cell types between day 3 and day 14 versus the baseline (day 0) (Supplementary Table 2, Supplementary Figure 5). We observed significant stress-induced dysregulation of two immune cell types, activated memory (but not resting) CD4 + T-cells and neutrophils, on day 14, the time point at which we also observed the strongest decline in the levels of the immune signaling molecules (Fig. 6). Activated memory CD4 + T-cell proportion showed a significant increase (two-tailed Wilcoxon signed-rank test $p$-value $=0.0008$ ), while the neutrophil fraction showed a significant decrease (two-tailed Wilcoxon signed-rank test $p$-value $=0.0019$ ) in response to stress. 

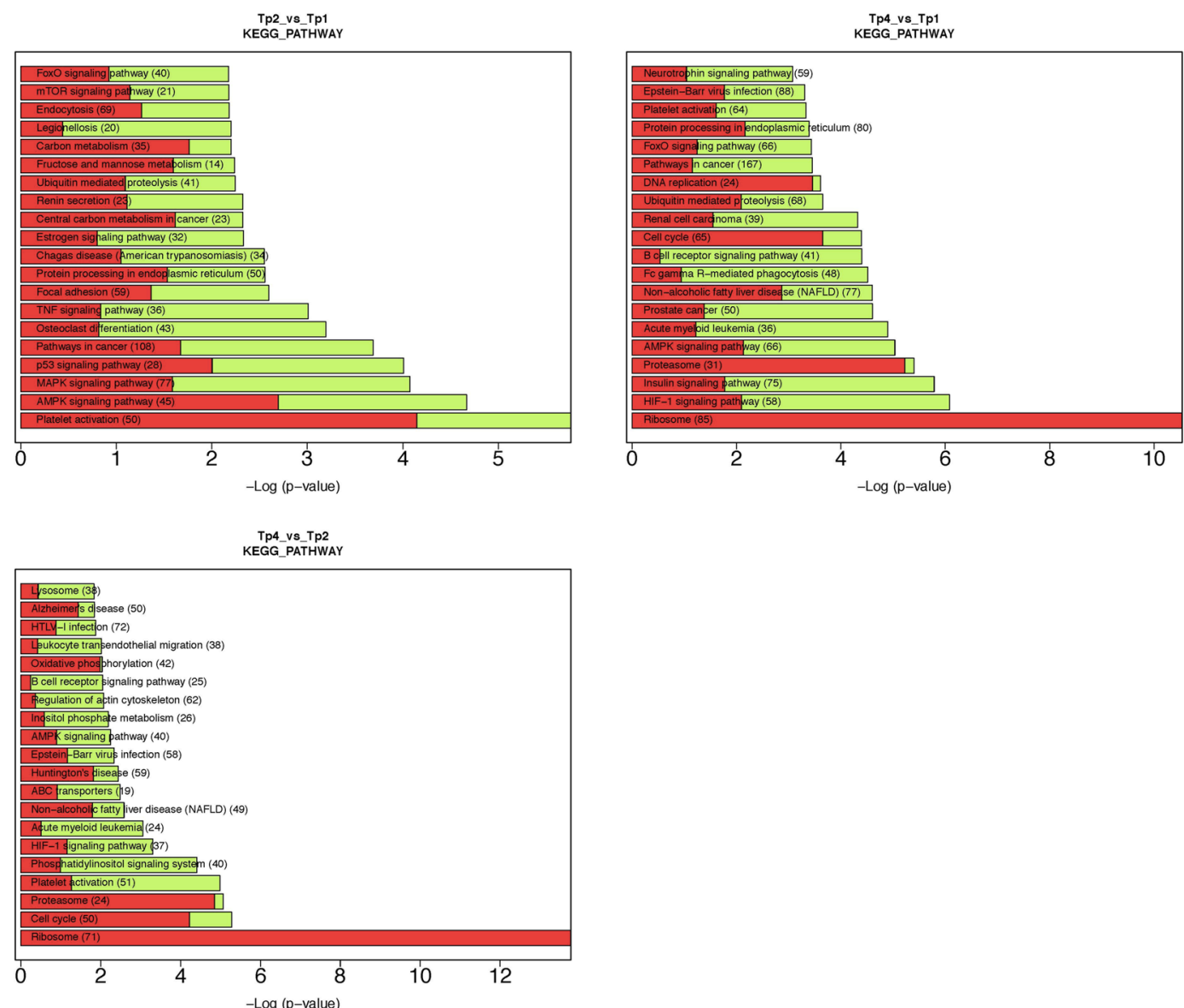

Figure 3. KEGG pathway enrichment analysis among genes dysregulated in response to stress based on the David tool.

\section{Discussion}

We have proposed and validated a natural model of PS, which leverages the routine quarantine procedure of wild-born vervets, to facilitate studies of responses to displacement from a natural social and physical environment under controlled conditions. The quarantine increased the accumulation of cortisol in hair, suggesting a sustained activation of the HPA axis concordant with chronic stress and thus supporting our model of PS. Stress can induce immunoprotective (e.g., promote wound healing and eliminate infections), immunopathological, and immunoregulatory effects depending on the context and the duration of the stressor ${ }^{36-40}$. We observed that, among the array of conventional health biomarkers of immune signalling, glycemic health and microbial translocation, nine cytokines, chemokines, and growth factors showed a rapid decline (reaching their lowest levels on day 14), followed by a varying rate of recovery to baseline levels. Given that both classical pro-inflammatory and anti-inflammatory molecules followed this decrease-recovery pattern, we conclude that it is consistent with the transient immunosuppressive effect of stress that is characteristic of chronic stress and followed by adaptation to the novel environment.

Chronically elevated cortisol levels and stress can induce hematological responses manifesting in quantitative alterations in key cell populations in the blood, for example, due to adrenal gland disease in Cushing syndrome or due to physical and psychological stressors in humans ${ }^{50,51}$. In vervets, PS differently modulated the proportions of immune cells during long-term stress (on day 14). An increase was observed in the activated CD $4+$ memory $\mathrm{T}$ cell proportion which are long-lived cells ${ }^{52}$ that may provide a protection against previously encountered pathogens. Our results are concordant with the observation that memory CD4 + T cells were positively linked to responses to a combination of aerobic exercise and heat stress in humans ${ }^{53}$. A PS-associated decrease was observed in the neutrophil fraction. Neutrophils are short-lived cells and the most abundant type of granulocytes acting as the first line of defense against infection as a part of the innate immune responses. They are involved in thrombotic processes, which suppress pathogen dissemination through coagulation ${ }^{54-56}$. The stress-related decrease of neutrophil ratios in the vervet model of PS suggests that the PS can render the exposed individuals more vulnerable to infections. In contrast to the stress related reduction of the neutrophil ratio during the PS in vervets, the neutrophil counts were positively associated with glucocorticoid levels in various vertebrates ${ }^{57}$ and psychosocial stress in humans $s^{50,58}$, suggesting that they may respond distinctively per species according to type of stressors and duration of exposure. In summary, our findings evidence that a strong impact of PS on immune functions in vervets. It caused immunosuppression characterised by a decrease in a number of important immune 
module-trait relationships

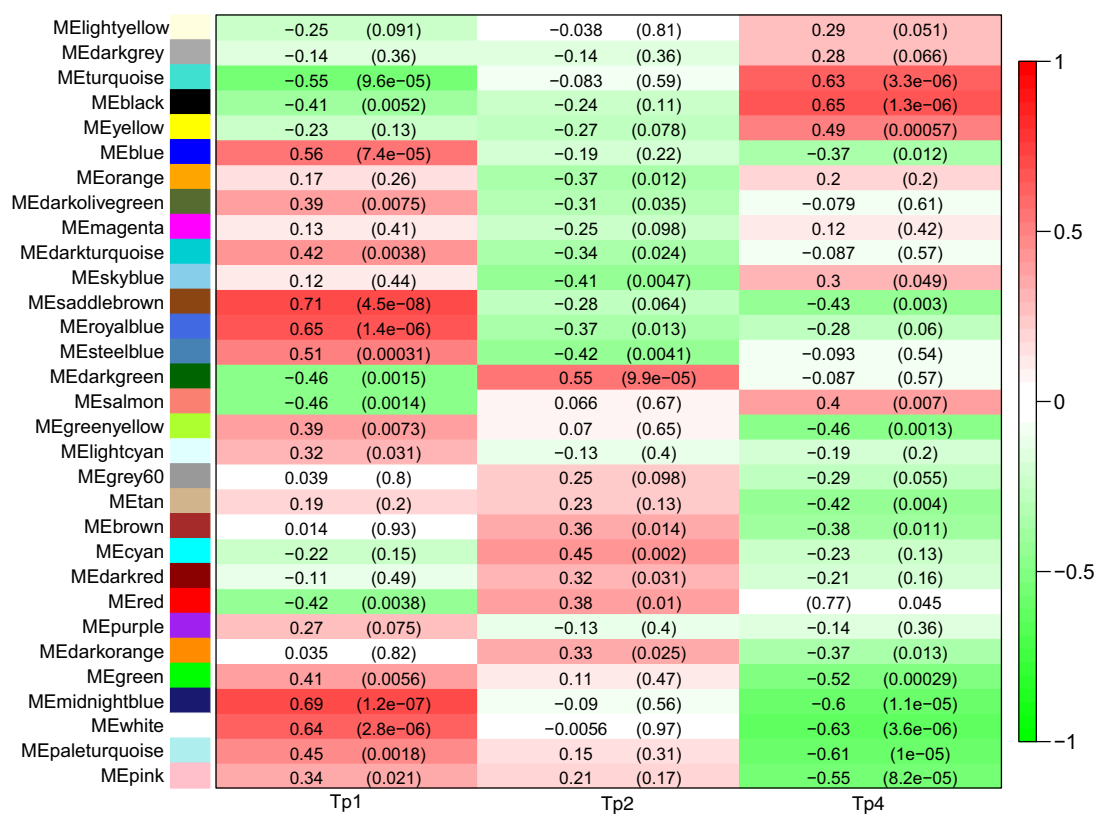

Figure 4. Module - time point correlation of the WGCNA modules. Each row corresponds to a module, while each column represents one time point: $\mathrm{Tp} 1$ i.e. baseline, $\mathrm{Tp} 2$ (day 3 ) and $\mathrm{Tp} 4$ (day 14). Correlation coefficients between the PC1 of a given module and specific time point are shown as color shades of each cell. The numeric value of correlation for each module-time point pair is also shown as numbers in each cell with a corresponding p-value beneath in parenthesis.

signaling molecules, and also appeared to impair neutrophil-associated innate immune functions. On the other hand, PS may also have a beneficial effect through increasing the activation of CD $4+$ memory T cells, which may protect against re-exposure to pathogens.

We observed a massive dysregulation of the blood transcriptome in response to PS in vervet, including genes that are involved in reactions to various types of stressors (such as chemical compounds) or relevant for stress-related diseases. Short-term exposure to stress (on day 3) impacted the regulatory pathways of cell survival or cell death, and KEGG pathways associated with the activation of genes crucial for blood coagulation and platelet activation that play a pivotal role in cardiovascular disease ${ }^{59}$. Psychological stress has long been known to alter cardiovascular function and platelet activity ${ }^{60,61}$ and our observation is consistent with the hypothesis that stress may potentiate coronary disease pathogenesis in part via the activation of blood platelets ${ }^{62}$. Short-term stress was also associated with the AMPK signaling pathway, which is involved in sensing cellular energy status, and MAPK pathway playing a key role in stress-induced depression ${ }^{63,64}$. The genes dysregulated during long-term stress (on day 14) are involved in the pathways of various homeostatic processes, including cell cycle and cancer regulation and insulin signaling. Multiple health-related conditions are known to be associated with genes, which showed strong dysregulation $(\log 2 \mathrm{FC}>3$ ) during stress exposure in vervet suggesting the relevance of the vervet model of PS to mechanisms linking human conditions with stress. For example, genes dysregulated upon short-term stress (on day 3 ) were linked to cardiovascular diseases (ACTA2), tumor processes (SIM2, POU6F2, ADAMTS12), inflammation and responses to microbes (PALM3, FLG2), reaction to oxidative stress (THSD4), and Alzheimer's disease $(C O B L)$; and genes dysregulated after long-term stress exposure (on day 14) included cardiomyopathies (TNNI3), oxidative stress (CTSE) and antiviral properties (IFIT1b), stress responses (GRIA1), and in neurobehavioral disorders (SLC6A2).

We demonstrated that the proposed paradigm of PS that models displacement and social isolation stressor in veret evoked significant stress manifested as increased cortisol production, mostly transient suppression of immune functions, and massive dysregulation of the blood transcriptome, including genes associated with a range of pathologies and phenotypes of cardiovascular and metabolic diseases, tumorigenesis, neurotransmission, and antimicrobial responses. However, we realize that stress-related responses, in particular the wide-spread effects on immune system functions (including, inflammatory biomarkers, blood cell composition, transcripts of genes involved in immune responses) we observed in vervets may be moderated by several variables that are unaccounted for here, including initial social rank and other behavioral attributes of the study animals. For example, personality traits were associated with transcriptional activity of pro-inflammatory genes in humans ${ }^{65}$ and social rank altered immune responses to infections in rhesus macaques ${ }^{66}$. Collecting baseline behavioral data in the natural habitat before the relocation to the captive setting could be considered in potential future studies. We envision that over time the vervet model of PS combined the longitudinal phenotyping based on minimally invasive procedures can facilitate well-powered investigations of the molecular, genomic and epigenetic mechanisms underlying shared and individual variable responses to stress. 

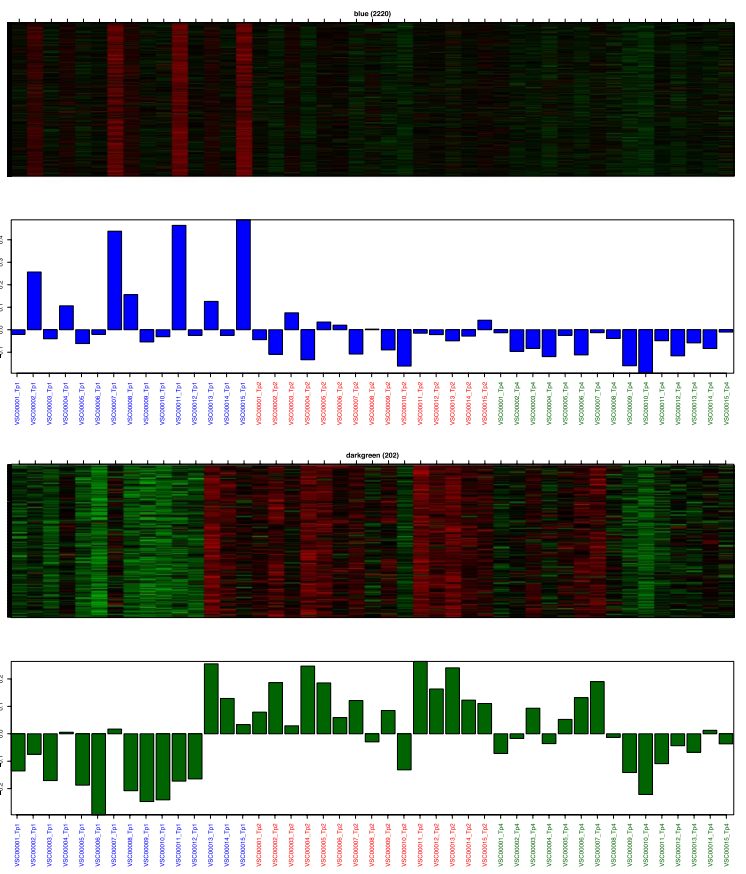

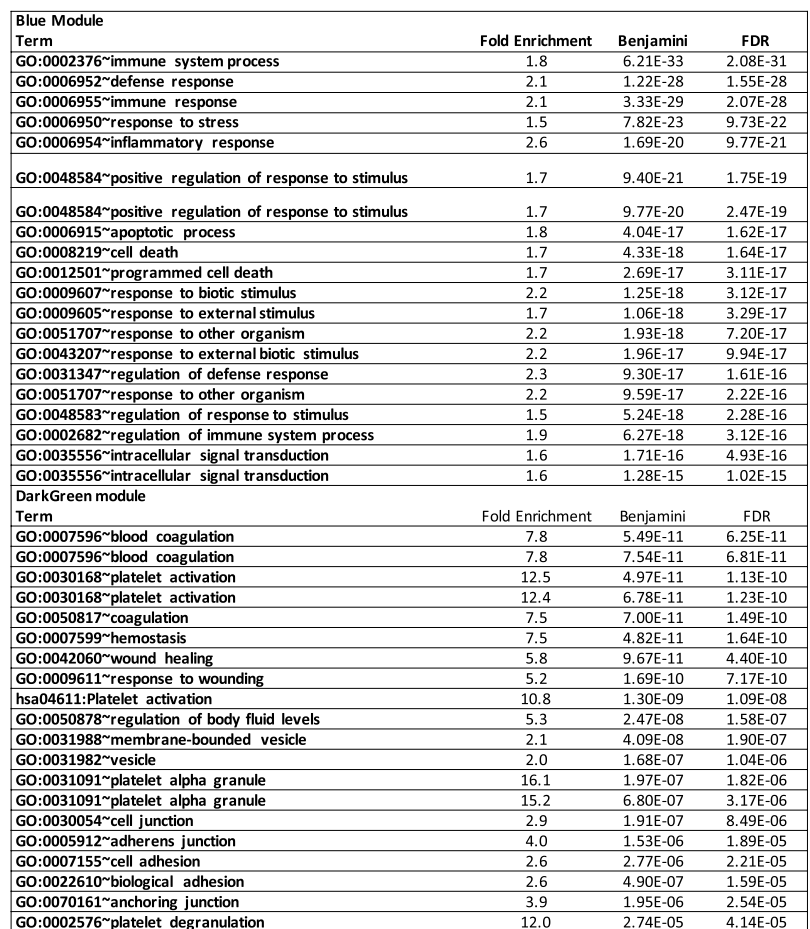

Figure 5. Eigengene (the 1st principal component) values of the blue and dark green WGCNA modules by increasing time points. Samples from 15 animals are organized by time points (first Tp1, then Tp 2, followed by TP4).

Materials and Methods

Neutrophils

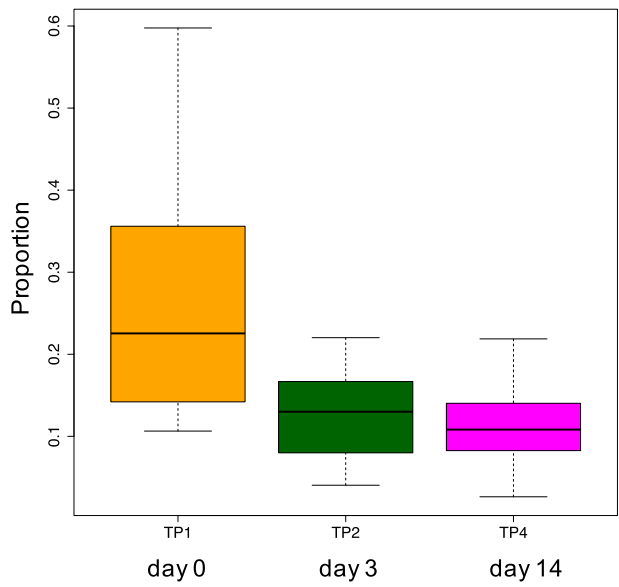

CD4 + T cells memory activated

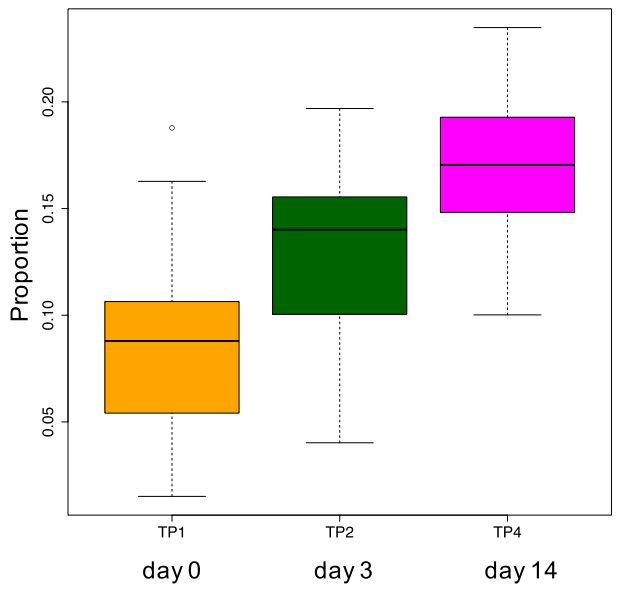

Figure 6. The alterations of neutrophil and activated memory CD4 + T-cell ratios across the experiment.

Ethics statement. All animals in this study were wild-born vervet monkeys from a local population on St. Kitts Island that were transferred to and housed in the local NHP facility of St. Kitts Biomedical Research Foundation (SKBRF) accredited by the Association for the Assessment and Accreditation of Laboratory Animal Care under accreditation number A4384-01. All procedures used in this study (including animal capture, transfer, quarantine, and sampling) were reviewed and approved by the Axion Research Foundation IACUC under protocol number AC14118 and the UCLA Office of Animal Research Oversight ARC under protocol number 2014-075-02 before implementation. All animal procedures and sample collections were in compliance with the US National Research Council's Guide for Care and Use of Laboratory Animals ${ }^{67}$ and meet or exceed all standards of the Public Health Service's Policy on the Humane Care and Use of Laboratory Animals ${ }^{68}$.

Study individuals. We acquired 15 juvenile male vervet monkeys from the wild vervet population on St. Kitts Island using a standardized, humane trapping procedure routinely used by the local primate facility (SKBRF) for animal acquisition ${ }^{28}$. The animals were transported to the SKBRF where they were subjected to conditions 
closely imitating a 28-day routine quarantine which, according to our hypothesis, represented significant PS. To longitudinally assess responses to PS, we collected biological samples and observational data over a 28-day period at six experimental time points (Tp) corresponding with a specific day (d) ( \pm 1 day) following capture. First, on day 0 (Tp1), baseline samples were immediately collected after trapping in the field before animals were transported to the SKBRF. Then, on day 3 (Tp2), day 7 (Tp3), day 14 (Tp4), Tp5 (day 21), and Tp6 (day 28), the animals were sampled under quarantine conditions (Supplementary Table 3 ).

On day 0 , animals were trapped and rapidly immobilized (within 2-3 minutes) with a sedative (intramuscularly administered ketamine $[10 \mathrm{mg} / \mathrm{kg}]$ mixed with xylazine $[2 \mathrm{mg} / \mathrm{kg}]$ ) and sampled within 15 minutes of capture, allowing us to significantly limit the impact of capture and sedation on gene expression and biochemical markers. The first sampling conducted in the field represented baseline conditions (day 0, Tp1). To minimize relatedness among the study subjects, only one individual per troop was acquired for this study (Supplementary Figure 6). Given the moderate sample size, we maximized the power by forming a homogeneous cohort with respect to developmental stage and sex, including only juvenile males. Co-trapped animals (not selected for our study) were intramuscularly administered the reversal agent atipamezole $(0.1 \mathrm{mg} / \mathrm{kg})$ to reverse the effects of the anesthetic and, after full recovery, were released back into the wild near the capture site so they could rejoin their troops.

Although, social status is a significant predictor of health and disease outcomes and regulator of stress perception and responses, including regulation of immune functions and their reactions to infection ${ }^{23,66,69-71}$, we were not able to assess pre-capture social rank in the troops in the wild, even though this variable could impact their accumulated life stress burden.

The animals selected for the PS study (15 juvenile males) were transported to the SKBRF immediately after the first sampling while they were still sedated. At arrival, the animals were placed in individual housing. Following the transfer, the animals were then sampled at four time points (day 3, 7, 14, 21, and 28, corresponding with Tp2Tp6). To perform sampling, the animals were briefly sedated with intramuscular dexmedetomidine $(0.05 \mathrm{mg} /$ $\mathrm{kg})$ mixed with midazolam $(0.3 \mathrm{mg} / \mathrm{kg})$. The sedative was reversed following the sampling with intramuscular atipamezole $(0.1 \mathrm{mg} / \mathrm{kg})$ mixed with flumazenil $(0.5 \mathrm{mg} / \mathrm{kg})$. The terminal sample was conducted on day 28 of the quarantine with the exception of animal VSC00015, which was sampled one day later.

Sample collection. We collected a wide array of biological samples. PaxGene RNA blood tube samples were collected on days 0 (Tp1), 7 (Tp3), and 14 (Tp4) as a source of total RNA for transcriptomic analysis; K2EDTA blood tube samples were collected on days 0 (Tp1), 7 (Tp3), 14 (Tp5), and 28 (Tp6) to measure inflammatory biomarkers; and hair samples were obtained on days 0 (Tp1) and 28 (Tp6) to perform long-term cortisol measurements. We limited survival blood collection to $1.25 \%(1.25 \mathrm{ml} / 100 \mathrm{~g})$ of each animal's current body weight per week according to the IACUC recommendations for preventing anemia and dehydration. To ensure that the blood collection limits were not exceeded, we collected only whole blood for RNAseq analysis and did not collect blood for plasma on day 3 (Tp2) and thus did not perform circulating biomarker measurements at this time point.

Cortisol levels measurements. Vervet hair is shed and regrown at a regular rate. On days 0 (Tp1) and 28 (Tp6), we collected hair samples, most of which were about $3 \mathrm{~cm}$. We assessed cortisol levels using methods previously validated for vervets ${ }^{31,72,73}$. Cortisol concentrations were assessed in full length hair, accounting for the weight of the sample (ranging from 15 to $42 \mathrm{mg}$ of hair). We did not include hair length in calculations since the results were normalized for weight. Assuming a similar hair growth rate as humans, the measured cortisol levels would be reflective of average cortisol secretion during the preceding three months.

Circulating inflammatory and glucose metabolism biomarkers. At five time points (days $0,7,14,21$, 28 [Tp1, Tp3-Tp6]), we measured an array of health-related biomarkers in blood plasma using biochemical measures previously validated for wild vervets ${ }^{74,75}$, including SCD14 (a soluble biomarker of microbial translocation), lipopolysaccharide (LPS), and a29-plex monkey panel of immune signaling molecules, including 15 cytokines (IL-1 $\beta$, IL-1RA, IL-2, IL-4, IL-5, IL-6, IL-10, IL-12, IL-15, IL-17, G-CSF, GM-CSF, IFN- $\gamma$, IP-10, TNF $\alpha$ ), 10 chemokines (CCL-11 [eotaxin], IL-8, CCL-2 [MCP-1], CCL-22 [MDC], MIF, CXCL-9 [MIG], CCL-3 [MIP-1 $\alpha$ ], CCL-4 [MIP-1ß], I-TAC, CCL-5 [RANTES]), and 4 growth factors (EGF, FGF-basic, HGF, and VEGF).

We also assessed circulating biomarkers of glucose metabolism, A1C hemoglobin, and total glycated hemoglobin GHb in whole blood at baseline day 0 (Tp1), day 14 (Tp4), and day 28 (Tp6); these measures reflect average blood glucose levels for the previous three months. For total glycated hemoglobin $(\mathrm{GHb})$ testing with calculated Alc levels, we used the Primate GHb/A1c Testing kit based on high performance liquid chromatography (HPLC boronate affinity) method (DTIL Laboratories, Inc.). Health-related biomarkers with five or more values $<0$ were discarded. Variables with fewer than five values $<0$ had negative values set to zero. A total of 16 health-related variables were analyzed (Table 1).

Transcriptome analysis using RNA-seq. Total blood RNA was extracted from PaxGene Blood RNA tubes (Preanalytix) using PAXgene Blood RNA Kit (Qiagen). Immediately after extraction, the RNA concentrations were measured with Nanodrop, and the integrity was assessed using a bioanalyzer (Supplementary Table 4). RNA integrity number (RIN) ranged from 7.5 to 9.1 , with an average value of 8.5 , and concentrations ranged from $11 \mathrm{ng} / \mathrm{ul}$ to $415 \mathrm{ng} / \mathrm{ul}$, with an average value of $127 \mathrm{ng} / \mathrm{ul}$.

We conducted RNA-seq in blood RNA samples on day 0 (Tp1), day 3 (Tp 2), and day 14 (Tp4). For cDNA library creation, we used the TruSeq Stranded RNA protocol and $100 \mathrm{ng}$ of RNA under RiboZero and Globin treatment (Illumina). We generated $120 \mathrm{bp}$-long paired-end sequencing reads using high-output flow cell lines in the HiSeq. 4000 sequencer (Illumina). Our dataset was deposited in the NCBI's Gene Expression Omnibus (GEO) under accession number GSE120900. 
Bioinformatic analysis. RNA-seq reads were aligned to the Chlorocebus sabaeus reference genome (Chlorocebus_sabeus 1.1 GCF_000409795.2) ${ }^{76}$ using the STAR aligner ${ }^{77}$. Fragment counts were derived using the HTSeq program ${ }^{78}$. Differential gene expression analysis was conducted using the Bioconductor packages. Count data were submitted to edgeR modeling with a negative binomial distribution ${ }^{79}$. In both cases, we used FDR-adjusted $\mathrm{p}$-values lower than 0.1. To characterize the correlation patterns among genes across the samples, we employed weighted gene correlation network analysis (WGCNA) ${ }^{80}$. Genes with a CPM $>0.5$ in at least 3 samples were selected for constructing the signed network. We used the Database for Annotation, Visualization and Integrated Discovery (DAVID) v6.8 to understand the biological functions of the genes that were differentially expressed or co-regulated in response to PS. We used available vervet gene annotations; however, they are not as rich and detailed as annotations for the human, so in the analysis we additionally used human annotations (including KEGG pathways) available in the David tool ${ }^{81}$.

To estimate the relative proportions of different cell types in the whole blood samples based on the bulk blood RNA-seq profiles, we applied CIBERSORT (for quantification of 22 immune cell types) ${ }^{49}$, which is an established computational method of deconvolution of transcriptomic data for inferring the cell composition of complex tissues.

\section{Data availability}

Our RNAseq dataset was deposited in the NCBI's Gene Expression Omnibus (GEO) under accession number GSE120900.

Received: 9 December 2019; Accepted: 3 February 2020;

Published online: 26 February 2020

\section{References}

1. Dimsdale, J. E. Psychological stress and cardiovascular disease. J. Am. Coll. Cardiol. 51, 1237-1246 (2008).

2. Hjemdahl, P. Stress and the metabolic syndrome: an interesting but enigmatic association. Circulation 106, 2634-2636 (2002).

3. Tamashiro, K. L., Sakai, R. R., Shively, C. A., Karatsoreos, I. N. \& Reagan, L. P. Chronic stress, metabolism, and metabolic syndrome. Stress 14, 468-474 (2011).

4. Song, H. et al. Stress related disorders and risk of cardiovascular disease: population based, sibling controlled cohort study. BMJ 365 , 11255 (2019).

5. Bacon, S. L. et al. Hemodynamic, hemostatic, and endothelial reactions to psychological and physical stress in coronary artery disease patients. Biol. Psychol. 71, 162-170 (2006).

6. Sareen, J. Posttraumatic stress disorder in adults: impact, comorbidity, risk factors, and treatment. Can. J. Psychiatry 59, 460-467 (2014).

7. Cohen, S., Janicki-Deverts, D. \& Miller, G. E. Psychological stress and disease. JAMA 298, 1685-1687 (2007).

8. Razzoli, M. et al. Social stress shortens lifespan in mice. Aging Cell e12778 (2018).

9. Hellhammer, D. H., Wüst, S. \& Kudielka, B. M. Salivary cortisol as a biomarker in stress research. Psychoneuroendocrinology 34, 163-171 (2009)

10. Meyer, J. S. \& Novak, M. A. Minireview: Hair cortisol: a novel biomarker of hypothalamic-pituitary-adrenocortical activity. Endocrinology 153, 4120-4127 (2012).

11. Shors, T. J. \& Servatius, R. J. The contribution of stressor intensity, duration, and context to the stress-induced facilitation of associative learning. Neurobiol. Learn. Mem. 68, 92-96 (1997).

12. Davis, E. P. \& Sandman, C. A. The timing of prenatal exposure to maternal cortisol and psychosocial stress is associated with human infant cognitive development. Child Dev. 81, 131-148 (2010).

13. Jung, C. M. et al. Acute effects of bright light exposure on cortisol levels. J. Biol. Rhythms 25, 208-216 (2010).

14. Bergendahl, M., Iranmanesh, A., Mulligan, T. \& Veldhuis, J. D. Impact of age on cortisol secretory dynamics basally and as driven by nutrient-withdrawal stress. J. Clin. Endocrinol. Metab. 85, 2203-2214 (2000).

15. Gaffey, A. E., Bergeman, C. S., Clark, L. A. \& Wirth, M. M. Aging and the HPA axis: Stress and resilience in older adults. Neurosci. Biobehav. Rev. 68, 928-945 (2016).

16. Grissom, N. \& Bhatnagar, S. Habituation to repeated stress: get used to it. Neurobiol. Learn. Mem. 92, 215-224 (2009).

17. Herman, J. P. Neural control of chronic stress adaptation. Front. Behav. Neurosci. 7, 61 (2013).

18. Phillips, K. A. et al. Why primate models matter. Am. J. Primatol. 76, 801-827 (2014).

19. Sapolsky, R. M. The influence of social hierarchy on primate health. Science 308, 648-652 (2005).

20. Watson, S. L., McCoy, J. G., Stavisky, R. C., Greer, T. F. \& Hanbury, D. Cortisol response to relocation stress in Garnett's bushbaby (Otolemur garnettii). Contemp. Top. Lab. Anim. Sci. 44, 22-24 (2005).

21. Davenport, M. D., Lutz, C. K., Tiefenbacher, S., Novak, M. A. \& Meyer, J. S. A rhesus monkey model of self-injury: effects of relocation stress on behavior and neuroendocrine function. Biol. Psychiatry 63, 990-996 (2008).

22. Schapiro, S. J. et al. Physiological and Welfare Consequences of Transport, Relocation, and Acclimatization of Chimpanzees (Pan troglodytes). Appl. Anim. Behav. Sci. 137, 183-193 (2012).

23. Tung, J. et al. Social environment is associated with gene regulatory variation in the rhesus macaque immune system. Proc. Natl. Acad. Sci. USA 109, 6490-6495 (2012).

24. Snyder-Mackler, N. et al. Social status alters chromatin accessibility and the gene regulatory response to glucocorticoid stimulation in rhesus macaques. Proc. Natl. Acad. Sci. USA 116, 1219-1228 (2019).

25. Meyer, J. S. \& Hamel, A. F. Models of stress in nonhuman primates and their relevance for human psychopathology and endocrine dysfunction. ILAR J. 55, 347-360 (2014).

26. Yen, I. H. \& Syme, S. L. The social environment and health: a discussion of the epidemiologic literature. Annu. Rev. Public Health 20, 287-308 (1999).

27. Cole, S. W. Social regulation of human gene expression: mechanisms and implications for public health. Am. J. Public Health 103(Suppl 1), S84-92 (2013).

28. Jasinska, A. J. et al. Systems biology of the vervet monkey. ILAR J. 54, 122-143 (2013).

29. Jasinska, A. J. et al. Genetic variation and gene expression across multiple tissues and developmental stages in a nonhuman primate. Nat. Genet. 49, 1714-1721 (2017).

30. Jasinska, A. J. Biological Resources for Genomic Investigation in the Vervet Monkey (Chlorocebus). Savanna Monkeys 16-28 (2019) https://doi.org/10.1017/9781139019941.002.

31. Fairbanks, L. A. et al. Heritability and genetic correlation of hair cortisol in vervet monkeys in low and higher stress environments. Psychoneuroendocrinology 36, 1201-1208 (2011). 
32. Fairbanks, L. A. et al. Environmental stress alters genetic regulation of novelty seeking in vervet monkeys. Genes Brain Behav. 10, 683-688 (2011)

33. Uno, H., Tarara, R., Else, J. G., Suleman, M. A. \& Sapolsky, R. M. Hippocampal damage associated with prolonged and fatal stress in primates. J. Neurosci. 9, 1705-1711 (1989).

34. Czoty, P. W., Gould, R. W. \& Nader, M. A. Relationship between social rank and cortisol and testosterone concentrations in male cynomolgus monkeys (Macaca fascicularis). J. Neuroendocrinol. 21, 68-76 (2009).

35. Dhabhar, F. S. Enhancing versus suppressive effects of stress on immune function: implications for immunoprotection and immunopathology. Neuroimmunomodulation 16, 300-317 (2009).

36. Segerstrom, S. C. \& Miller, G. E. Psychological stress and the human immune system: a meta-analytic study of 30 years of inquiry. Psychol. Bull. 130, 601-630 (2004).

37. Steptoe, A., Hamer, M. \& Chida, Y. The effects of acute psychological stress on circulating inflammatory factors in humans: a review and meta-analysis. Brain Behav. Immun. 21, 901-912 (2007).

38. Dhabhar, F. S. Effects of stress on immune function: the good, the bad, and the beautiful. Immunol. Res. 58, 193-210 (2014).

39. Dhabhar, F. S. \& Mcewen, B. S. Acute Stress Enhances while Chronic Stress Suppresses Cell-Mediated Immunityin Vivo:A Potential Role for Leukocyte Trafficking. Brain Behav. Immun. 11, 286-306 (1997).

40. Liu, Y.-Z., Wang, Y.-X. \& Jiang, C.-L. Inflammation: The Common Pathway of Stress-Related Diseases. Frontiers in Human Neuroscience vol. 11 (2017).

41. Bailey, M. T. Psychological Stress, Immunity, and the Effects on Indigenous Microflora. Adv. Exp. Med. Biol. 874, 225-246 (2016).

42. Lafuse, W. P. et al. Exposure to a Social Stressor Induces Translocation of Commensal Lactobacilli to the Spleen and Priming of the Innate Immune System. J. Immunol. 198, 2383-2393 (2017).

43. Liu, R. T. The microbiome as a novel paradigm in studying stress and mental health. Am. Psychol. 72, 655-667 (2017).

44. Pouwer, F., Kupper, N. \& Adriaanse, M. C. Does emotional stress cause type 2 diabetes mellitus? A review from the European Depression in Diabetes (EDID) Research Consortium. Discov. Med. 9, 112-118 (2010).

45. Hilliard, M. E. et al. Stress and A1c Among People with Diabetes Across the Lifespan. Curr. Diab. Rep. 16, 67 (2016).

46. Deshpande, A. D., Harris-Hayes, M. \& Schootman, M. Epidemiology of diabetes and diabetes-related complications. Phys. Ther. 88, 1254-1264 (2008).

47. Pani, L. N. et al. Effect of Aging on A1C Levels in Individuals Without Diabetes: Evidence from the Framingham Offspring Study and the National Health and Nutrition Examination Survey 2001-2004. Diabetes Care 31, 1991-1996 (2008).

48. Maranville, J. C. et al. Interactions between glucocorticoid treatment and cis-regulatory polymorphisms contribute to cellular response phenotypes. PLoS Genet. 7, e1002162 (2011).

49. Newman, A. M. et al. Robust enumeration of cell subsets from tissue expression profiles. Nat. Methods 12, 453-457 (2015).

50. Heidt, T. et al. Chronic variable stress activates hematopoietic stem cells. Nat. Med. 20, 754-758 (2014).

51. Castinetti, F., Morange, I., Conte-Devolx, B. \& Brue, T. Cushing's disease. Orphanet J. Rare Dis. 7, 41 (2012).

52. Westera, L. et al. Closing the gap between T-cell life span estimates from stable isotope-labeling studies in mice and humans. Blood 122, 2205-2212 (2013).

53. Gannon, G. A., Rhind, S., Shek, P. N. \& Shephard, R. J. Naive and memory T cell subsets are differentially mobilized during physical stress. Int. J. Sports Med. 23, 223-229 (2002).

54. Tak, T., Tesselaar, K., Pillay, J., Borghans, J. A. M. \& Koenderman, L. What's your age again? Determination of human neutrophil half-lives revisited. J. Leukoc. Biol. 94, 595-601 (2013).

55. Massberg, S. et al. Reciprocal coupling of coagulation and innate immunity via neutrophil serine proteases. Nat. Med. 16, 887-896 (2010).

56. Sivanandham, R. et al. Neutrophil extracellular trap production contributes to pathogenesis in SIV-infected nonhuman primates. J. Clin. Invest. 128, 5178-5183 (2018).

57. Davis, A. K., Maney, D. L. \& Maerz, J. C. The use of leukocyte profiles to measure stress in vertebrates: a review for ecologists. Funct. Ecol. 22, 760-772 (2008).

58. Nishitani, N. \& Sakakibara, H. Association of psychological stress response of fatigue with white blood cell count in male daytime workers. Ind. Health 52, 531-534 (2014)

59. Willoughby, S., Holmes, A. \& Loscalzo, J. Platelets and cardiovascular disease. Eur. J. Cardiovasc. Nurs. 1, 273-288 (2002).

60. Koudouovoh-Tripp, P. \& Sperner-Unterweger, B. Influence of mental stress on platelet bioactivity. World J. Psychiatry 2, 134-147 (2012).

61. Fioranelli, M. et al. Stress and Inflammation in Coronary Artery Disease: A Review Psychoneuroendocrineimmunology-Based. Front. Immunol. 9, 2031 (2018).

62. Brydon, L., Magid, K. \& Steptoe, A. Platelets, coronary heart disease, and stress. Brain Behav. Immun. 20, 113-119 (2006)

63. Iio, W., Matsukawa, N., Tsukahara, T., Kohari, D. \& Toyoda, A. Effects of chronic social defeat stress on MAP kinase cascade. Neurosci. Lett. 504, 281-284 (2011).

64. Zhu, S. et al. Unpredictable chronic mild stress induces anxiety and depression-like behaviors and inactivates AMP-activated protein kinase in mice. Brain Res. 1576, 81-90 (2014).

65. Vedhara, K. et al. Personality and gene expression: Do individual differences exist in the leukocyte transcriptome? Psychoneuroendocrinology 52, 72-82 (2015).

66. Snyder-Mackler, N. et al. Social status alters immune regulation and response to infection in macaques. Science 354, 1041-1045 (2016).

67. National Research Council, Division on Earth and Life Studies, Institute for Laboratory Animal Research \& Committee for the Update of the Guide for the Care and Use of Laboratory Animals. Guide for the Care and Use of Laboratory Animals: Eighth Edition. (National Academies Press, 2011).

68. PHS Policy on Humane Care and Use of Laboratory Animals | OLAW. https://olaw.nih.gov/policies-laws/phs-policy.htm.

69. Sapolsky, R. M. Social Status and Health in Humans and Other Animals. Annu. Rev. Anthropol. 33, 393-418 (2004).

70. Baum, A., Garofalo, J. P. \& Yali, A. M. Socioeconomic status and chronic stress. Does stress account for SES effects on health? Ann. N. Y. Acad. Sci. 896, 131-144 (1999).

71. Larrieu, T. et al. Hierarchical Status Predicts Behavioral Vulnerability and Nucleus Accumbens Metabolic Profile Following Chronic Social Defeat Stress. Curr. Biol. 27, 2202-2210.e4 (2017).

72. Laudenslager, M. L., Jorgensen, M. J., Grzywa, R. \& Fairbanks, L. A. A novelty seeking phenotype is related to chronic hypothalamicpituitary-adrenal activity reflected by hair cortisol. Physiol. Behav. 104, 291-295 (2011).

73. Laudenslager, M. L., Jorgensen, M. J. \& Fairbanks, L. A. Developmental patterns of hair cortisol in male and female nonhuman primates: lower hair cortisol levels in vervet males emerge at puberty. Psychoneuroendocrinology 37, 1736-1739 (2012).

74. Ma, D. et al. SIVagm infection in wild African green monkeys from South Africa: epidemiology, natural history, and evolutionary considerations. PLoS Pathog. 9, e1003011 (2013).

75. Ma, D. et al. Factors associated with siman immunodeficiency virus transmission in a natural African nonhuman primate host in the wild. J. Virol. 88, 5687-5705 (2014).

76. Warren, W. C. et al. The genome of the vervet (Chlorocebus sabaeus). Genome Res. 25, 1921-1933 (2015).

77. Dobin, A. et al. STAR: ultrafast universal RNA-seq aligner. Bioinformatics 29, 15-21 (2013). 
78. Anders, S., Pyl, P. T. \& Huber, W. HTSeq-a Python framework to work with high-throughput sequencing data. Bioinformatics 31, 166-169 (2015).

79. Robinson, M. D., McCarthy, D. J. \& Smyth, G. K. edgeR: a Bioconductor package for differential expression analysis of digital gene expression data. Bioinformatics 26, 139-140 (2010).

80. Langfelder, P. \& Horvath, S. WGCNA: an R package for weighted correlation network analysis. BMC Bioinformatics 9, 559 (2008).

81. Dennis, G., Sherman, B. T., Hosack, D. A. et al. DAVID: Database for Annotation, Visualization, and Integrated Discovery. Genome Biol 4, R60, https://doi.org/10.1186/gb-2003-4-9-r60 (2013).

\section{Acknowledgements}

This research was funded by the NIH grants R21OD017959 to A.J.J. We acknowledge the support of the NINDS Informatics Center for Neurogenetics and Neurogenomics (P30 NS062691) and NIH grants 1R01HL123096 and R01DK113919 to I.P. and R01HL117715 to I.P. J.C.L. was involved in this research through the UCLA SRP99 and SRP199 programs. We thank Dr. Stephanie Groman, Mr. Oliver Pess Morton, Mr. O’Neal Whattley and the staff of SKBRF for assisting with the animal procedures and sample collection.

\section{Author contributions}

A.J.J., I.P., T.H., C.B., M.N. and J.C.L. produced the data. A.J.J. and G.C. analyzed the data. A.J.J., N.B.F. and J.D.J. designed the study. A.J.J. and J.D.J. wrote the paper. All authors reviewed the final draft.

\section{Competing interests}

The authors declare no competing interests.

\section{Additional information}

Supplementary information is available for this paper at https://doi.org/10.1038/s41598-020-59934-z.

Correspondence and requests for materials should be addressed to A.J.J.

Reprints and permissions information is available at www.nature.com/reprints.

Publisher's note Springer Nature remains neutral with regard to jurisdictional claims in published maps and institutional affiliations.

Open Access This article is licensed under a Creative Commons Attribution 4.0 International License, which permits use, sharing, adaptation, distribution and reproduction in any medium or format, as long as you give appropriate credit to the original author(s) and the source, provide a link to the Creative Commons license, and indicate if changes were made. The images or other third party material in this article are included in the article's Creative Commons license, unless indicated otherwise in a credit line to the material. If material is not included in the article's Creative Commons license and your intended use is not permitted by statutory regulation or exceeds the permitted use, you will need to obtain permission directly from the copyright holder. To view a copy of this license, visit http://creativecommons.org/licenses/by/4.0/.

(c) The Author(s) 2020 